\title{
A comparison of automated RF circuit design methodologies: online vs. offline passive component design
}

\author{
F. Passos, Student Member, IEEE, E. Roca, R. Castro-Lopez and F. V. Fernandez, Member, IEEE
}

\begin{abstract}
In this paper, surrogate modeling techniques are applied for passive component modeling. These techniques are exploited to develop and compare two alternative strategies for automated radio-frequency (RF) circuit design. The first one is a traditional approach where passive components are designed during the optimization stage. The second one, inspired on bottom-up circuit design methodologies, builds passive component Pareto-optimal fronts (POFs) prior to any circuit optimization. Afterwards, these POFs are used as an optimized library from where the passive components are selected. This work exploits the advantages of evolutionary computation algorithms in order to efficiently explore the circuit design space, and the accuracy and efficiency of surrogate models to model passive components.
\end{abstract}

Index Terms-design methodologies, radio-frequency, multiobjective optimizations, low noise amplifiers, surrogate modeling

\section{INTRODUCTION}

$\mathrm{T}$ HE design of complex systems on chip (SoC) in nanometer technologies is becoming immeasurably difficult due to its growing complexity. This fact, in combination with the many critical trade-offs in SoCs, such as, power consumption, operation speed, chip area, reliability, etc., and the tightening time-to-market constraints, result in a productivity gap for SoC design [1]. In order to bridge this gap, new design methodologies that allow designers to reduce the time-to-market and design circuits with the best trade-offs have to be devised. SoCs are nowadays gradually evolving towards mixed-signal designs, embedding analog, radiofrequency (RF), mixed-signal and digital blocks. For digital circuits, different design methodologies and electronic design automation (EDA) tools are available that help designers at performing hierarchical system design, hardware-software codesign and place \& route [1]. However, there is a scarcity of methodologies and tools that can assist the analog/RF designer.

Paper submitted on March 15, 2018. Revised on June 6, 2018. This work was supported in part by the P12-TIC-1481 Project (funded by Junta de Andalucia) and in part by the TEC2013-45638-C3-3-R and TEC2016-75151C3-3-R Project (funded by the Spanish Ministry of Science, Innovation and Universities and ERDF). F. Passos acknowledges the same ministry for supporting his research activity through the grant BES-2014-068216.

All authors are with the Instituto de Microelectrónica de Sevilla, CNMCSIC and Universidad de Sevilla, Seville, Spain (corresponding author: F. V. Fernandez, e-mail: Francisco.Fernandez@imse-cnm.csic.es).
In the emerging telecommunications market, evolving towards $5 \mathrm{G}$ and with a huge growth of wireless services, the need for design solutions with very demanding performance specifications is going to dramatically increase in the foreseeing years. Due to the complex trade-offs in RF circuits (e.g., noise vs. gain in a low noise amplifier), it is possible to understand that the design of RF systems is a complicated task that must be assisted by EDA tools and smart RF design methodologies. In an ideal scenario, the user would only stipulate the desired specifications for his/her system and the tool would automatically generate the IC. However, for complex systems such as RF circuits, such a tool is far from becoming reality.

Optimization-based techniques are gathering much attention in order to assist the RF designer into designing circuits [2][11]. These methodologies apply algorithms that are able to perform a wide design space exploration to find optimal designs. The idea behind these methodologies is that the designer choses the circuit specifications, selects the topology and runs an optimization that returns the circuit sizing (e.g., widths and lengths of transistors). In this type of design methodologies, single- or multi-objective optimization algorithms can be used depending on whether the designer is searching for a single design [5], or a Pareto-optimal front (POF) that represents the best trade-offs between several performances [6].

An important aspect in any RF circuit design methodology is how passive components are designed, especially passive components whose performances are negatively impacted by parasitics, such as integrated inductors. Several strategies have been proposed in the literature [7]-[11]. Some approaches use a reduced set of inductors provided by the foundries. Other approaches integrate accurate electromagnetic (EM) simulations of the inductors within the circuit optimization loop [7], which would be the most accurate method, but also the most computationally expensive. Thus, the optimization loop would become very time consuming and the efficiency of the process would dramatically fade. On the other extreme of the efficiency-accuracy trade-off is the use of lumped-element models (like the $\pi$-model) [8],[9]. However, these models are not accurate enough, especially at high frequencies [12].

Improving the efficiency-accuracy trade-off has become the holy grail in RF design methodologies. With this goal, a new strategy, inspired on circuit-level bottom-up design 
methodologies, was introduced in [11]. Typically, in bottomup design methodologies, the designer starts from the lowest level (e.g., cell level) and then proceeds up the hierarchy to eventually reach the system level (e.g., sensor interface or RF front-end), ensuring, therefore, that all levels are feasible and avoiding re-design cycles. In this type of design methodologies, multi-objective optimization algorithms are commonly used; therefore, the information passed to the upper level is not a single design solution but rather a POF, representing the best trade-offs available for a given circuit (e.g., phase noise vs. power consumption in a voltage controlled oscillator) [6],[10],[11]. The methodology in [11] exploits the accuracy of EM simulations and palliates the impact of the high computational times by decoupling inductor design from circuit design, hence avoiding any EM simulation during the circuit optimization loop. The idea consists on generating an inductor POF before any circuit optimization. This method allows the designer to build an optimized inductor library with inductors that are not designed specifically for a given circuit, and can, therefore, be used in any circuit. This methodology is based on the assumption that if the designer generates an inductor POF before the circuit optimization, the best inductor designs will be available in the POF and no other inductor would be capable of improving circuit performances. This assumption would be strictly correct if the true inductor POF were generated. However, a practical implementation is necessarily limited to an approximation to the true POF with a finite number of points.

Recently, surrogate models, have proven to be an efficient, but accurate, alternative to EM simulations in order to design integrated inductors [12],[13]. Surrogate modelling is an engineering technique used to predict system performances that cannot be easily measured or efficiently simulated. Surrogate models can accurately compute the behavior of any given system while still being computationally cheap to evaluate [14]. Therefore, in this work, the capabilities of surrogate models are exploited in order to efficiently and accurately evaluate inductors within a circuit optimization loop. In this way, the inductor design can be performed concurrently with the circuit design, as shown in [15] with surrogate models of the inductor S-parameters. Furthermore, surrogate models can also enable an alternative design strategy, similar to that reported in [11], as shown in [16] for a voltage-controlled oscillator. This paper explores which is the most advantageous RF design methodology: an online strategy, where the inductors are designed during the circuit optimization stage, or an offline strategy, where the inductors are optimized before any circuit optimization. By using the same type of inductor modeling strategy in both cases, it is reasonable to assume that the accuracy in terms of inductor performance evaluation is similar and it is possible to assess the influence of the offline/online design methodology in the quality and efficiency of the design results ${ }^{1}$.

\footnotetext{
${ }^{1}$ Notice that the terminology offline/online refers here to the instant of time in which the inductors are designed. A similar terminology has also been used in the surrogate modeling literature to refer to the construction of surrogate models in advance or along the optimization process.
}

To this end, the rest of this paper is structured as follows. Section II briefly presents both design methodologies and their basic ingredients, such as the optimization algorithm and the surrogate modeling approach. Section III develops both methodologies for the design of a low noise amplifier (LNA) and exhaustively compares the results. Finally, in Section IV conclusions are drawn.

\section{OfFLine AND OnLINE Design STRATEGIES}

Basic ingredients for the implementation of the design methodologies are EM simulators, RF circuit simulators, optimization tools and generation of surrogate models. The EM and RF simulators used in this work are commercially available; hence, the two latter elements will be discussed in this Section together with the description of both design methodologies.

\section{A. Basics of optimization algorithms}

RF circuit design problems can be formulated as optimization problems, mathematically:

$$
\begin{array}{cc}
\text { minimize } f(x) ; & f(x) \in R^{m} \\
\text { subject to } g(x) \leq 0 ; & g(x) \in R^{k} \\
x \in \Omega &
\end{array}
$$

where $f(x)$ is a vector of $m$ objective functions, $g(x)$ is a vector of $k$ constraints and $x$ is a vector of $n$ design variables on the search space $\Omega$.

When only one performance is minimized $(m=1)$, the problem can be solved with a single-objective optimization algorithm. In case that assessment of trade-offs between two or more conflicting objectives is desired $(m>1)$, then, a multiobjective optimization algorithm can be applied.

In the multi-objective case, a solution $a$ is said to constraindominate solution $b$ if and only if $a$ has a smaller constraint violation than $b$, or, if all constraints are met, $f_{i}(a) \leq f_{i}(b)$, for every $i \in\{1, \ldots, m\}$ and $f_{j}(a)<f_{j}(b)$ for at least an index $j \in\{1, \ldots, m\}$. A point $y \in \Omega$ is Pareto-optimal if it is not dominated by any other point in $\Omega$. The set of all Paretooptimal points is named the Pareto-optimal set and the corresponding points in the feasible objective space forms the Pareto-optimal front (POF). The goal of any multi-objective optimization algorithm is to obtain a set of uniformly distributed solutions as close as possible to the true POF.

In this work, as we are mainly interested in obtaining POFs to compare online strategies vs. offline bottom-up design strategies, only a multi-objective optimization algorithm is used: NSGA-II [17]. The RF circuit design methodologies considered in this paper do not exploit any special characteristic of NSGA-II; therefore, it could be replaced by any other multi-objective algorithm.

\section{B. Online design methodology}

The online RF circuit design strategy is illustrated in Fig. 1. The circuit design problem is addressed via an optimization 


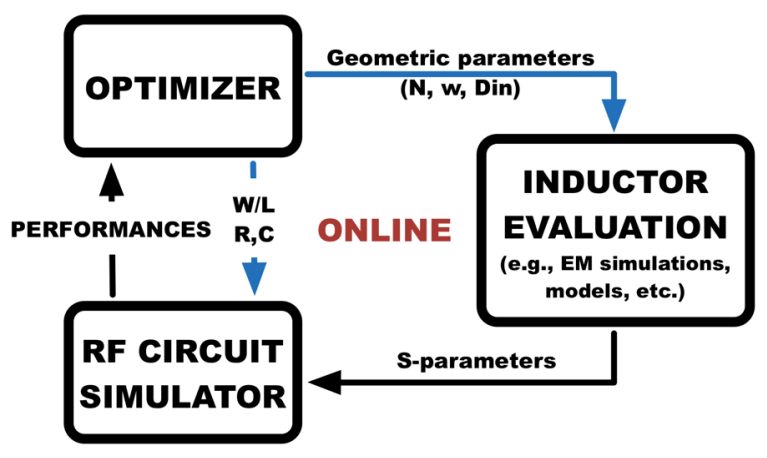

Fig. 1. Online design strategy.

loop involving the evaluation of a number of candidate solutions at each iteration.

Commercial RF circuit simulators provide the maximum accuracy for the evaluation of the performances of such solutions, while still being efficient enough (typically in the range of seconds) to be embedded within an optimization loop. RF circuit simulators provide accurate circuit performance evaluations as long as accurate models are available for each component used (e.g., transistors, etc.). This is the case for transistors, resistors and capacitors; however, inductors constitute a bottleneck due to its distributed effects and parasitics. Analytical and physical models (e.g., $\pi$-models) have been widely used but they are not very accurate. As modern RF circuit simulators are able to handle devices modeled by S-parameter files, a potentially accurate strategy is to model the inductors by using them. The inductor geometric parameters can be mapped to these S-parameters by using the surrogate modeling strategy described in Section II.D.

\section{Offline bottom-up design methodology}

The offline bottom-up design methodology is illustrated in Fig. 2. The bottom-up strategy is implemented by hierarchically partitioning the synthesis problem in two levels. At the lowest level, NSGA-II is used to generate the best trade-offs between inductor performances (i.e., a POF) involving inductance, quality factor and area. Surrogate models of the inductance and quality factor are used to generate the inductor front at this level. At the upper level, a circuit optimization loop is performed, using a similar strategy to that in Section II.B, but instead of using geometrical variables of the inductors as search variables, inductors can only be selected among those of the lowest level inductor POF. Each point in the inductor POF represents a certain inductance and quality factor; however, these characteristics are not appropriate for accurate RF circuit simulation. Hence, an S-parameter description generated by the surrogate modeling strategy in Section II.D is used, but, this time, only applied to the inductors in the POF.

\section{Surrogate modeling of integrated inductors}

As discussed above, both design methodologies are supported by surrogate modeling techniques. In the last few years, surrogate models are being used to model passive devices that are computationally expensive to evaluate (e.g., transmission lines, antennas, inductors, etc.) [13],[18]-[20]. Some approaches to develop surrogate models available in the literature are based on artificial neural networks, others in support vector machines, parametric macromodels or Kriging functions [14]. In this work, surrogate models are created based on ordinary Kriging functions. Basically, Kriging is an interpolation method for which the interpolated values are modeled by a Gaussian process. A clear overview of Kriging models and more insight on their mathematical properties can be found in [21].

A two-step strategy is proposed in [13] to accurately model inductance and quality factor at any frequency of interest. In this approach, a surrogate model of the self-resonance frequency (SRF) of the inductors is trained with a set of inductor samples, and models of inductance and quality factor are trained only with those inductors of the set with SRF above the working frequency. Any time an estimation of inductance and quality factor for new values of the inductor's geometric parameters is required, its SRF is first estimated by using the SRF surrogate model. If the estimated value is not above the working frequency, the inductor is skipped as it would yield a useless design. Otherwise, the surrogate models of inductance and quality factor are used to estimate their values.

The strategy presented in [13] is devoted to estimate inductance and quality factor and was originally conceived to generate Pareto fronts of inductor performances. However,
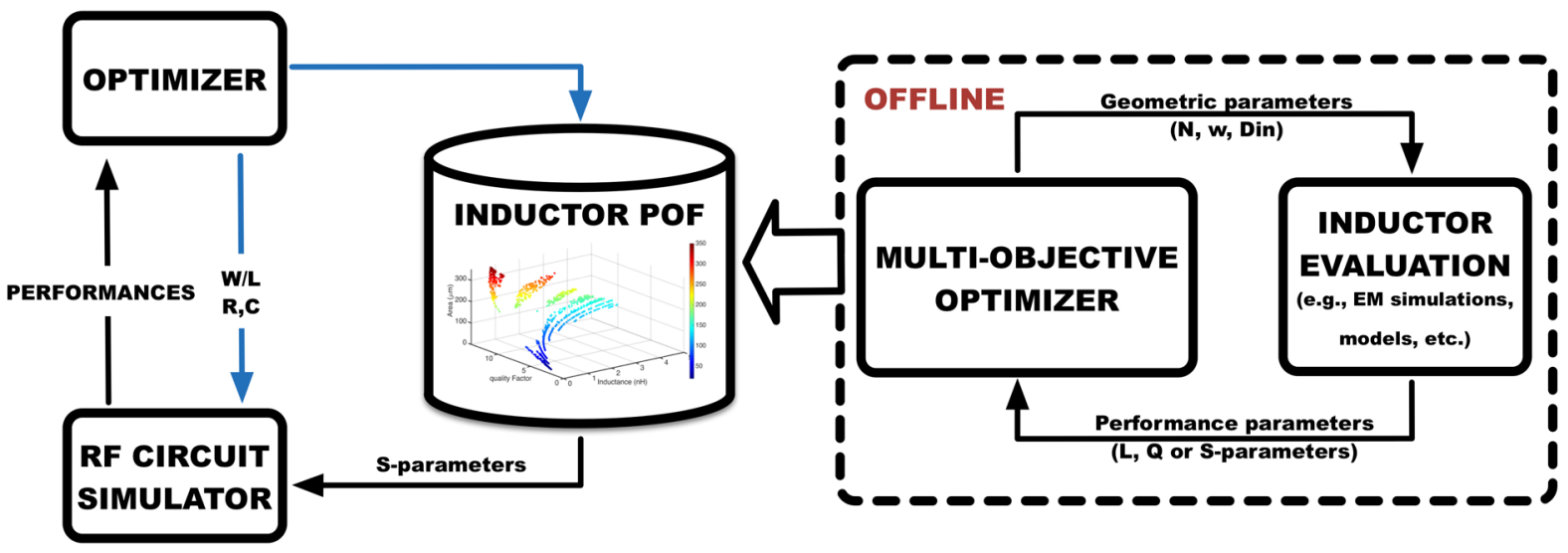

Fig. 2. Offline bottom-up design strategy. 
inductance and quality factor are not appropriate for RF circuit level simulation since they cannot be easily mapped into an accurate simulatable model. Therefore, instead of directly modeling the inductor performance parameters, such as inductance $L$ and quality factor $Q$, the inductor's S-parameters are modeled. In this way, the inductor can be accurately described over a wide range of frequencies and its description is included in circuit level simulation by using RF circuit simulators, such as SpectreRF [22]. In this work, eight different models were built in order to model the real and imaginary part of each S-parameter, and also the inductors were separately modeled by the number of turns $N$. All models in this paper were created using a zero-order polynomial regression model and a Gaussian correlation model. The models were trained using $800 \mathrm{EM}$-simulated inductors whose geometries were sampled within the design space shown in Table I using the Quasi-Monte Carlo (QMC) technique. The developed surrogate models were first validated against another 240 inductors, simulated electromagnetically, in order to check its accuracy. The geometric parameters of the 240 inductors were also sampled using the $\mathrm{QMC}$ technique. Table II shows the mean relative errors for the predicted value of the S-parameters with respect to EM simulations. It can be seen that some errors in the real or imaginary parts of the Sparameters (e.g., 1.89\%) are significantly larger than those on inductance and quality factor at the last two columns in Table II. However, these higher errors are due to the small values of the S-parameters of some inductor samples, small absolute errors being reflected into relatively high percentages. However, when these S-parameters are combined to get $L$ and $Q$ values, the model errors are always below $1 \%$. Moreover, the models are evaluated in just a matter of milliseconds. It can be concluded that the surrogate model used in this work is very accurate and efficient; therefore, it may be considered for an accurate RF circuit optimization.

TABLE I

DESIGN VARIABLES FOR THE INDUCTORS USED IN THE LNA OPTIMIZATION.

\begin{tabular}{cc|cc|cc}
\hline \hline \multicolumn{2}{c}{ Number of turns $N$} & \multicolumn{2}{c}{ Inner diameter $\boldsymbol{D}_{\text {in }}(\boldsymbol{\mu m})$} & \multicolumn{2}{c}{ Turn width $\boldsymbol{W}(\boldsymbol{\mu m})$} \\
\hline Min-Max & Grid & Min-Max & Grid & Min-Max & Grid \\
\hline $1-8$ & 1 & $10-300$ & 0.05 & $5-25$ & 0.05 \\
\hline \hline
\end{tabular}

\section{APPLICATION CIRCUIT}

\section{A. Inductor POF generation}

For the offline bottom-up design strategy, an inductor POF has to be generated prior to any circuit optimization. Therefore, an optimization using NSGA-II was performed. The search space is presented in Table I (the same one is used in both online and offline strategies). The optimization problem has three objectives: maximize quality factor and inductance at the working frequency, while minimizing the area. The inductors were subjected to a set of constraints (used to guarantee that the inductors are in the flat bandwidth zone [23]), defined as:

$$
\left\{\begin{array}{c}
\text { area }<400 \mu m \times 400 \mu m \\
\left|\frac{L_{@ W F}-L_{@(W F+0.05 G H z)}}{L_{@ W F}}\right|<0.01 \\
\left|\frac{\left.L_{@ W F}-L_{@(W F-0.05 G H z}\right)}{L_{@ W F}}\right|<0.01 \\
\left|\frac{L_{@ W F}-L_{@ 0.1 \mathrm{GHz}}}{L_{@ W F}}\right|<0.05 \\
\left.Q_{@(W F+0.05 \mathrm{GHz}}\right)-Q_{@ W F}>0
\end{array}\right.
$$

where $\mathrm{L}_{@ W F}$ and $\mathrm{Q}_{@ W F}$ are the inductance and quality factor at the working frequency. The inductance and quality factor at any frequency can be easily obtained from the S-parameters [24].

The design quality of the LNA will be eventually influenced by the density of the points at the inductor POF; hence, the number of points must be sufficiently high. The POF obtained for 1,000 individuals and 80 generations of asymmetric octagonal inductors for a working frequency of $2.5 \mathrm{GHz}$ can be observed in Fig. 3. The generation of this POF takes only ten minutes of CPU, so it is more than reasonable to use this population size. It should be noticed that this POF is independent of the circuit design objectives and constraints and therefore can be reused for any other circuit design at the same frequency band.

\section{B. LNA design strategy}

LNAs are used in RF circuits in order to amplify the low power signals coming from the antenna while trying not to degrade the signal-to-noise ratio. For the experiments in this paper, the source-degenerated topology in Fig. 4 will be used.

TABLE II

MEAN RELATIVE ERROR IN \% FOR THE PREDICTED VALUES OF S-PARAMETERS WITH RESPECT TO EM SIMULATIONS

\begin{tabular}{ccccccccccc}
\hline \hline $\boldsymbol{N}$ & $\boldsymbol{R e}\left\{\boldsymbol{S}_{\mathbf{1 1}}\right\}$ & $\boldsymbol{I m}\left\{\boldsymbol{S}_{\mathbf{1 1}}\right\}$ & $\boldsymbol{R e}\left\{\boldsymbol{S}_{\mathbf{2 1}}\right\}$ & $\boldsymbol{I m}\left\{\boldsymbol{S}_{\mathbf{2 1}}\right\}$ & $\boldsymbol{R e}\left\{\boldsymbol{S}_{\mathbf{1 2}}\right\}$ & $\boldsymbol{I m}\left\{\boldsymbol{S}_{\mathbf{1 2}}\right\}$ & $\boldsymbol{R e}\left\{\boldsymbol{S}_{\mathbf{2 2}}\right\}$ & $\boldsymbol{I m}\left\{\boldsymbol{S}_{\mathbf{2 2}}\right\}$ & $\boldsymbol{L}(\mathbf{\%})$ & $\boldsymbol{Q}(\mathbf{\%})$ \\
\hline $\mathbf{1}$ & 1.61 & 0.88 & 0.00 & 0.04 & 0.00 & 0.04 & 1.37 & 0.85 & 0.09 & 0.38 \\
\hline $\mathbf{2}$ & 1.54 & 0.96 & 0.01 & 0.05 & 0.01 & 0.05 & 1.20 & 0.76 & 0.11 & 0.55 \\
\hline $\mathbf{3}$ & 0.46 & 0.14 & 0.02 & 0.05 & 0.02 & 0.05 & 0.43 & 0.14 & 0.11 & 0.21 \\
\hline $\mathbf{4}$ & 0.34 & 0.09 & 0.05 & 0.05 & 0.05 & 0.05 & 0.27 & 0.10 & 0.12 & 0.34 \\
\hline $\mathbf{5}$ & 0.17 & 0.09 & 0.66 & 0.05 & 0.63 & 0.05 & 0.16 & 0.09 & 0.26 & 0.38 \\
\hline $\mathbf{6}$ & 0.16 & 0.08 & 1.00 & 0.04 & 1.04 & 0.04 & 0.14 & 0.07 & 0.40 & 0.79 \\
\hline $\mathbf{7}$ & 0.06 & 0.03 & 0.21 & 0.02 & 0.19 & 0.02 & 0.06 & 0.05 & 0.44 & 0.44 \\
\hline $\mathbf{8}$ & 0.34 & 0.12 & 1.89 & 0.07 & 1.89 & 0.07 & 0.31 & 0.14 & 0.24 & 0.71 \\
\hline \hline
\end{tabular}




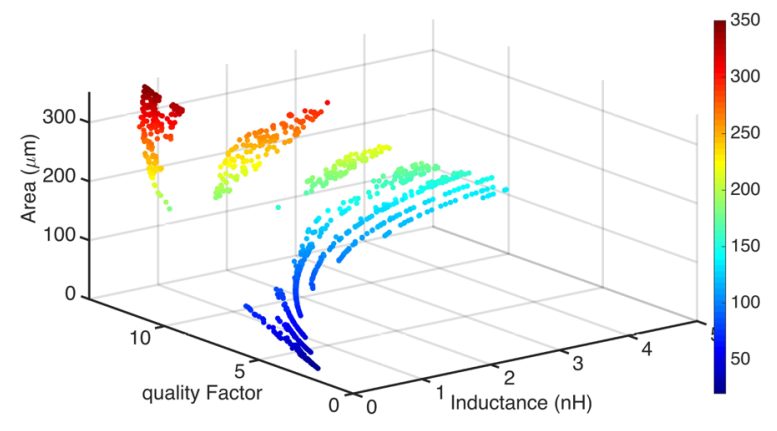

Fig. 3. Inductor POF obtained with NSGA-II.

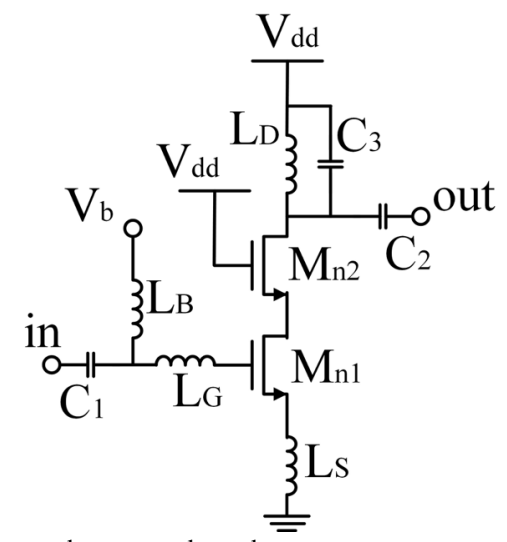

Fig. 4. LNA source-degenerated topology.

The LNA has several important performance parameters that must be considered during the design process:

- NF: noise figure, which accounts for the unwanted additional noise that the LNA injects in the input signal.

- $S_{21}$ : gain.

- $P_{\mathrm{DC}}$ : power consumption.

- IIP3: third-order input intercept point, which accounts for the nonlinear performance of the LNA.

- $S_{11}$ : input matching coefficient.

- $S_{22}$ : output matching coefficient.

- $K$ : Rollet stability factor. If this factor is smaller than 1 the LNA is potentially unstable.

- Area occupation, extremely important as it is directly related to the manufacturing cost in IC technologies.

In both design strategies compared in this work (see Fig. 1 and Fig. 2), an optimization algorithm is linked with an RF circuit simulator. However, in the online strategy (Fig. 1) a surrogate model is used to evaluate new inductors for each individual LNA during the optimization stage. For the offline strategy (Fig. 2), an inductor POF is used and the inductors are selected from the POF, for which the inductors must be identified with indices [11]. The indexing process is performed by grouping the inductors based on its performance parameters (inductance and quality factor) and assigning two coordinates to the inductors according to their performance values. These coordinates can later be used as search variables in the LNA optimization process.

The optimization process and the methodology itself is completely independent of the fabrication technology and the LNA topology used and only requires the following user inputs:

1. Desired circuit performances (optimization objectives and constraints)

2. Circuit design variables (e.g., transistors widths and lengths)

Regarding the circuit performances, the RF electrical simulator SpectreRF is used to evaluate each LNA. The power consumption $P_{\mathrm{DC}}$ is extracted from a DC analysis. An Sparameter analysis provides the gain, input and output matching, whereas a noise analysis provides the $N F$ of the LNA. The Rollett stability factor, $K$, can be calculated from the S-parameters of the LNA:

$K=\frac{1-\left|S_{11}\right|^{2}-\left|S_{22}\right|^{2}+\left|S_{11} S_{22}-S_{12} S_{21}\right|^{2}}{2\left|S_{12}\right|\left|S_{21}\right|}$

The area occupation is not accurately known until the circuit layout is performed. However, it is realistic to estimate that the layout area will be proportional to the sum of the areas of the individual devices (e.g., transistors, inductor and capacitors in Fig. 4). Therefore, the use of this sum as estimation of the circuit area is a valid approximation for comparison of different candidate solutions.

The inclusion of IIP3 in automated design methodologies usually takes long computation times since the input power must be swept to calculate the IIP3. Additionally, the IIP3 calculation is difficult since linearity severely deteriorates with higher values of the input power. Therefore, selecting the best input power points to determine IIP3 is not trivial and varies for each sized circuit. The method used in this work in order to efficiently include IIP3 in the optimization process assumes that IIP3 is directly related with $P_{\mathrm{DC}}$. Therefore, once $P_{\mathrm{DC}}$ is calculated for a given sized circuit, IIP3 can be calculated for an extrapolated input power well below $P_{\mathrm{DC}}$ so that the LNA is in the linear region [11]. We have determined that $60 \mathrm{~dB}$ below $P_{\mathrm{DC}}$ guarantees a linear relation between input and output power. Using this method, the computation time for IIP3 measurement is considerably reduced and it is feasible to include it within the LNA optimization loop.

Regarding the LNA design variables, these are shown in Tables I and III. For the online design strategy, where the inductors are optimized online, there are 18 design variables (those in Table III plus three variables per inductor corresponding to Table I). For the offline strategy, where the inductors are selected from the POF, the number of variables is reduced to 14 ( 8 in Table III and two indices per inductor for their identification in the POF).

TABLE III

DESIGN VARIABLES FOR THE LNA OPTIMIZATION (OTHER THAN INDUCTORS)

\begin{tabular}{ccccc}
\hline \hline Variables & Min & Max & Grid \\
\hline Transistor widths: $\boldsymbol{w}_{M 1}, \boldsymbol{w}_{M 2}(\boldsymbol{\mu m})$ & 100 & 600 & 5 \\
\hline Transistor lengths: $\boldsymbol{l}_{\boldsymbol{M} 1}, \boldsymbol{l}_{\boldsymbol{M} 2}(\boldsymbol{\mu \mathrm { m }})$ & \multicolumn{3}{c}{ Fixed at 0.35} \\
\hline $\boldsymbol{V}_{\boldsymbol{b}}(\mathrm{V})$ & 0 & 1.5 & 0.001 \\
\hline $\boldsymbol{C}_{\boldsymbol{1}}, \boldsymbol{C}_{2}, \boldsymbol{C}_{3}(\mathrm{pF})$ & 0.1 & 5 & 0.001 \\
\hline \hline
\end{tabular}




\section{Optimization results}

In this section, five different design examples are used to compare both strategies. For each example, the constraints and objectives are shown in Table IV. The examples with two objectives used 300 individuals and 200 generations, whereas 1,000 individuals and 300 generations were used for the 3objective cases. All optimizations in this work were performed for the LNA topology shown in Fig. 4 in a $0.35 \mu \mathrm{m}-\mathrm{CMOS}$ technology intended to operate at the frequency band of 2.4$2.5 \mathrm{GHz}$, with a supply voltage $V_{d d}=2.5 \mathrm{~V}$.

The first optimization example was performed with two objectives: maximize $S_{21}$ and minimize area. The POF of both online and offline optimizations can be observed in Fig. 5. It is possible to conclude that the offline strategy achieves much lower areas for the same values of gain, and even achieves higher values of gain.

The second optimization example also has two objectives: minimize area and noise figure. The POFs provided by the optimization algorithm can be seen in Fig. 6. In this design example, it is possible to observe that the offline strategy allows relatively lower noise figures and lower areas. In the third example, where the minimization of both area and power consumption is desired, the offline strategy achieves designs with much lower area for the same power consumption (see Fig. 7).

The last two examples were performed with 3 objectives. In example 4 the objectives are gain maximization and minimization of area and noise figure. The obtained POF is shown in Fig. 8. Similar values of noise figure and gain are obtained in the offline case with lower areas. Example 5 has the maximization of the gain and the minimization of the area and the power consumption as objectives and the resulting Pareto front is shown in Fig. 9. Better values of gain are obtained in the offline case for similar values of area and power consumption. Despite the quality difference found in the fronts shown in Figs. 5 to 9, it can be argued that no conclusion can be definitively formulated. Evolutionary algorithms are known to introduce a degree of randomness in the search process. This means that two different executions will provide different results and no conclusion can be derived from a single run. To address this potential problem, each algorithm was executed ten times. The ten fronts for the offline and online experiments of example 2 above are shown in Fig. 10. It can be observed that despite the variability of the results, the offline results are clearly better. However, the

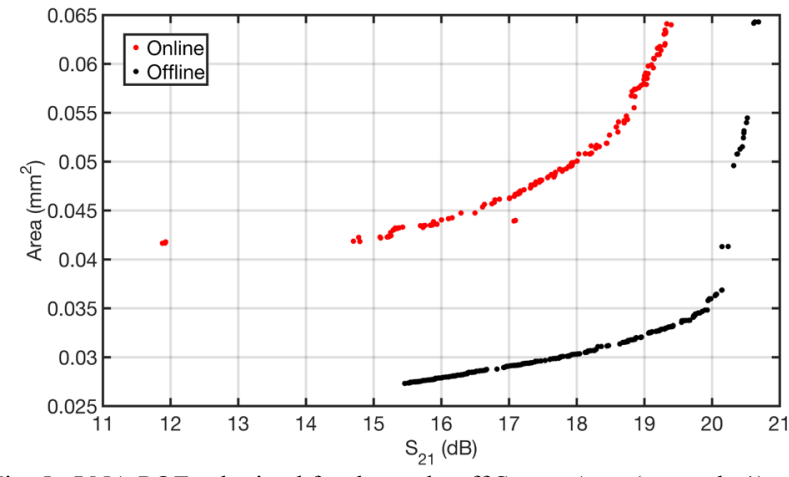

Fig. 5. LNA POFs obtained for the trade-off $\mathrm{S}_{21}$ vs. Area (example 1).

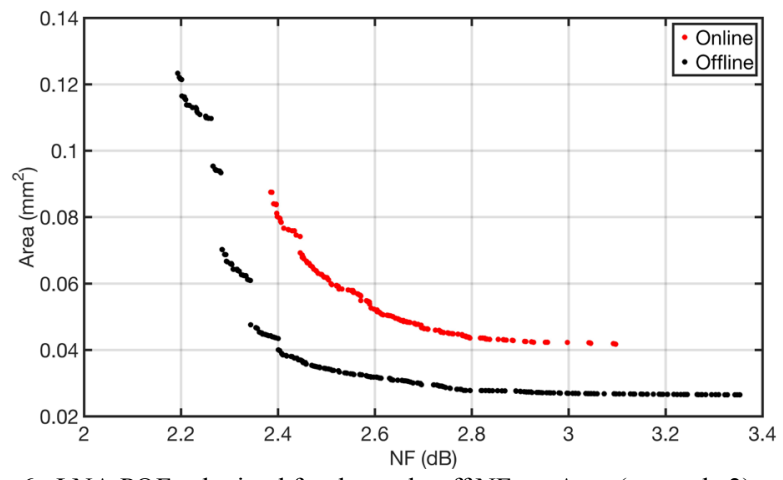

Fig. 6. LNA POFs obtained for the trade-off NF vs. Area (example 2).

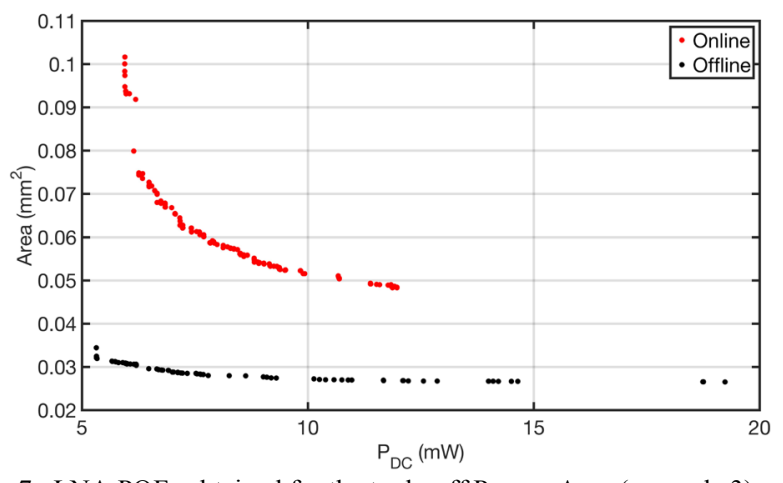

Fig. 7. LNA POFs obtained for the trade-off $P_{D C}$ vs. Area (example 3).

comparison is not always easy to visualize and it becomes extremely difficult in the experiments with three objectives.

In order to properly compare the POFs, some comparison metrics must be used. Among the several metrics available in the literature, hypervolume will be used in this paper [25]. The hypervolume accounts for convergence (closeness of the

TABLE IV

DESIRED SPECIFICATION FOR THE LNA OPTIMIZATION USING NSGA-II FOR FIVE DIFFERENT EXAMPLES.

\begin{tabular}{|c|c|c|c|c|c|}
\hline Performance & Example 1 & Example 2 & Example 3 & Example 4 & Example 5 \\
\hline$S_{11}$ & $<-10 \mathrm{~dB}$ & $<-10 \mathrm{~dB}$ & $<-10 \mathrm{~dB}$ & $<-10 \mathrm{~dB}$ & $<-10 \mathrm{~dB}$ \\
\hline$S_{22}$ & $<-10 \mathrm{~dB}$ & $<-10 \mathrm{~dB}$ & $<-10 \mathrm{~dB}$ & $<-10 \mathrm{~dB}$ & $<-10 \mathrm{~dB}$ \\
\hline$S_{21}$ & Maximize $(>10 \mathrm{~dB})$ & $>10 \mathrm{~dB}$ & $>10 \mathrm{~dB}$ & Maximize $(>10 \mathrm{~dB})$ & $\operatorname{Maximize}(>10 \mathrm{~dB})$ \\
\hline$K$ & $>1$ & $>1$ & $>1$ & $>1$ & $>1$ \\
\hline$N F$ & $<3 \mathrm{~dB}$ & Minimize & $<3.5 \mathrm{~dB}$ & Minimize & $<3.5 \mathrm{~dB}$ \\
\hline $\boldsymbol{P}_{\mathrm{DC}}$ & $<25 \mathrm{~mW}$ & $<25 \mathrm{~mW}$ & Minimize & $<10 \mathrm{~mW}$ & Minimize \\
\hline IIP3 & $>-10 \mathrm{dBm}$ & $>-10 \mathrm{dBm}$ & $>-10 \mathrm{dBm}$ & $>-10 \mathrm{dBm}$ & $>-10 \mathrm{dBm}$ \\
\hline Inductors & \multicolumn{5}{|c|}{ Constraints in eq. (2) for $\mathrm{WF}=2.45 \mathrm{GHz}$} \\
\hline Area $\left(\mu \mathrm{m}^{2}\right)$ & Minimize & Minimize & Minimize & Minimize & Minimize \\
\hline
\end{tabular}




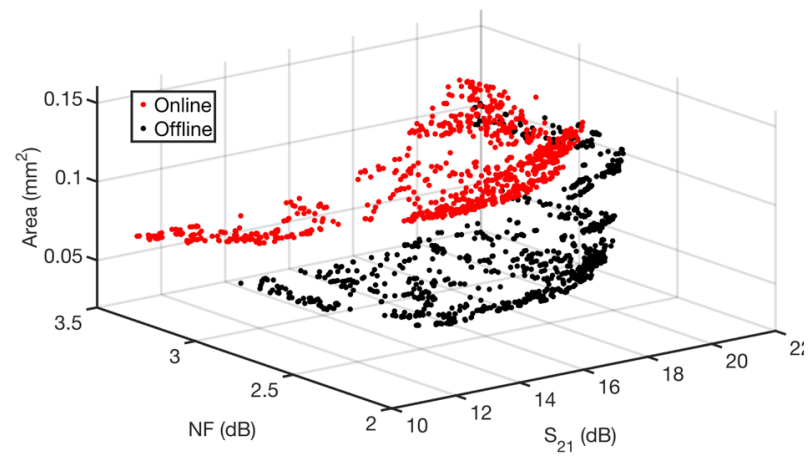

Fig. 8. LNA POFs obtained for the trade-off $\mathrm{S}_{21} \mathrm{vs}$. NF vs. Area (example 4).

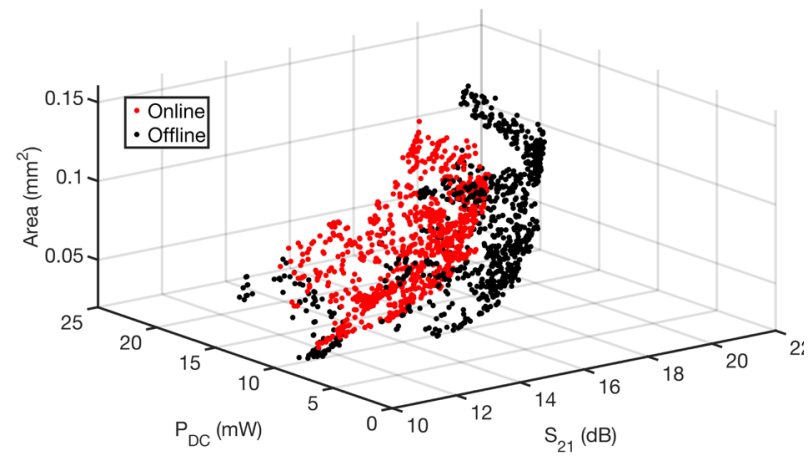

Fig. 9. LNA POFs obtained for the trade-off $S_{21}$ vs. Area vs. $P_{D C}$ (example 5).

solutions to the true Pareto front) and diversity (extension of the approximated Pareto front and uniformity of the solutions in it). The hypervolume is calculated as the sum of the hypercubes determined by each point of the approximated POF and a reference point. A common approach for the reference point is to select the worst values of all objectives. As our goal is to compare the POFs generated with two different methodologies, and the hypervolume metric depends on the selected reference point, the same reference point is used in both cases. Higher hypervolume values are associated to better convergence to the true POF and better diversity of the solutions along the POF. Table V presents the statistical results of the ten executions. It can be seen that the offline strategy obtains higher hypervolume values for all examples, proving once again the benefits of the offline strategy.

The reasons for the better performance of the offline strategy can be rooted to the inductor search space. The online circuit design strategy searches in the complete design space of the inductor geometric variables whereas the offline strategy only explores a search space of inductors with optimal trade-offs of inductor performances. Whereas the inductor search space of the online circuit design case, according to the data in Table I, amounts to $8 \times(300-10) \times 20 \times(25-5) \times 20=$ 18560000 possible solutions, the inductor search space in the offline case is limited to the 1,000 inductors of the Pareto front. Not only the search space is much smaller, but those

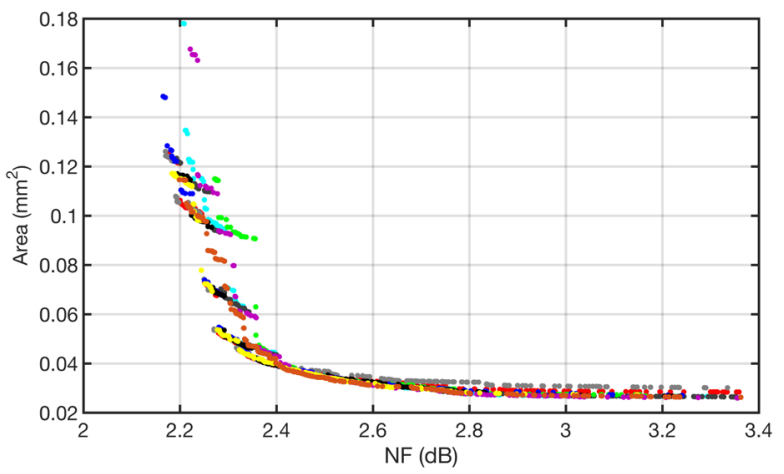

(a)

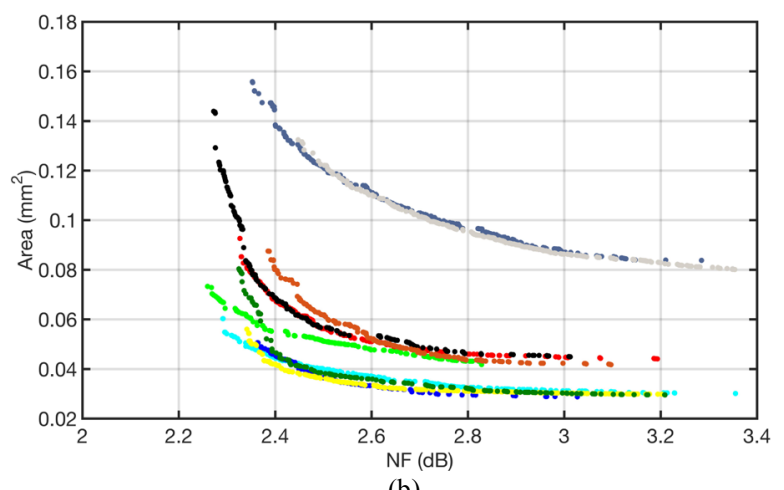

(b)

Fig. 10. POFs obtained in 10 runs of example 2 for the (a) offline and (b) online cases

1,000 inductors are already optimal. As a consequence, the optimization process in the online case is much harder. To illustrate this, the hypervolume of the ten executions of the online and offline optimization processes of example 2 have been monitored through the generations, as shown in Fig. 11. The same reference point was used for both configurations and all generations. It can be checked that not only the offline optimization process yields much better results but the optimization converges faster.

It could also be argued that the surrogate model error could be behind the worse performance of the online optimization. Although the average errors in Table II are low, a large error in a region of the search space visited during the optimization process might deviate the optimization to a worse region. Addressing this concern by comparison of each surrogate model evaluation to an EM simulation would be impossible. If we consider that the examples above imply evaluating thousands of individuals $(300 \times 200$ LNAs in the first three examples and $1000 \times 300$ in the other two), each containing four inductors, and that each experiment was repeated 10 times, we would need millions of EM simulations. Therefore, the selected feasible strategy was to perform two different tests. First, two inductors were selected from every final solution of a random execution of all online optimization examples above. This implies the EM simulation of 5,800 inductors that allows to assess the best, worst and mean relative errors of the surrogate models in Table VI. Then, two inductors of a randomly selected solution of each generation of a randomly selected execution of each online optimization 
TABLE V

HYPER VOLUME OBTAINED FOR THE DIFFERENT EXPERIMENTAL EXAMPLES.

\begin{tabular}{c|ccc|c|cccc}
\hline \hline \multirow{2}{*}{ Experiments } & \multicolumn{9}{|c|}{ Offline } & \multicolumn{4}{c}{ Online } \\
\cline { 2 - 9 } & Best & Mean & Worst & Standard deviation & Best & Mean & Worst & Standard deviation \\
\hline Example 1 & 1.0929 & 1.0498 & 0.9907 & 0.0401 & 1.0038 & 0.7569 & 0.3287 & 0.2337 \\
\hline Example 2 & 0.1671 & 0.1638 & 0.1557 & 0.0036 & 0.1530 & 0.1290 & 0.0761 & 0.0284 \\
\hline Example 3 & 0.0021 & 0.0020 & 0.0017 & 0.0001 & 0.0020 & 0.0015 & 0.0007 & 0.0004 \\
\hline Example 4 & 2.0532 & 2.0377 & 2.0246 & 0.0110 & 1.9849 & 1.5421 & 0.8710 & 0.3932 \\
\hline Example 5 & 0.0292 & 0.0288 & 0.0284 & 0.0002 & 0.0256 & 0.0214 & 0.0122 & 0.0045 \\
\hline \hline
\end{tabular}

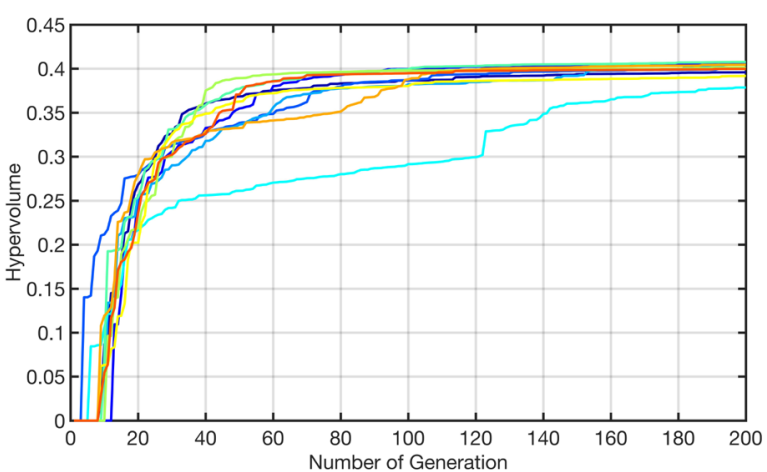

(a)

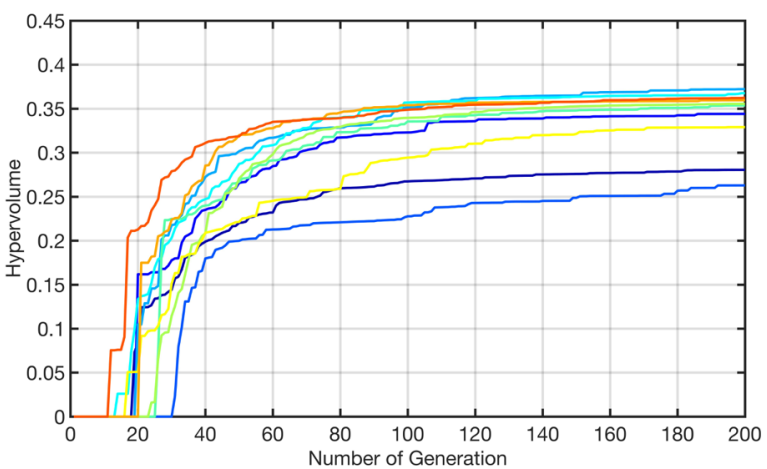

(b)

Fig. 11. Evolution of the hypervolume along the number of generations for the (a) offline and (b) online cases of example 2.

TABLE VI

ERROR FOR 5800 RANDOM INDUCTORS OF THE FINAL SOLUTIONS.

\begin{tabular}{c|ccc|ccc}
\hline \hline \multirow{2}{*}{ Experiment } & \multicolumn{2}{|c|}{ Error in inductance (\%) } & \multicolumn{3}{c}{ Error in quality factor (\%) } \\
\cline { 2 - 7 } & Best & Mean & Worst & Best & Mean & Worst \\
\hline Example 1 & 0.0002 & 0.0849 & 0.3057 & 0.0011 & 0.2171 & 0.7997 \\
\hline Example 2 & 0.0034 & 0.1134 & 0.7106 & 0.0104 & 0.1811 & 0.4944 \\
\hline Example 3 & 0.0014 & 0.1911 & 0.4543 & 0.0011 & 0.1367 & 0.3765 \\
\hline Example 4 & 0.0002 & 0.1278 & 0.7246 & 0.0011 & 0.2515 & 0.7997 \\
\hline Example 5 & 0.0001 & 0.0883 & 0.1951 & 0.0011 & 0.2076 & 0.5590 \\
\hline \hline
\end{tabular}

TABLE VII

ERROR FOR 4780 RANDOM INDUCTORS OF DIFFERENT GENERATIONS.

\begin{tabular}{c|ccc|ccc}
\hline \hline \multirow{2}{*}{ Experiment } & \multicolumn{2}{|c|}{ Error in inductance (\%) } & \multicolumn{2}{c}{ Error in quality factor (\%) } \\
\cline { 2 - 7 } & Best & Mean & Worst & Best & Mean & Worst \\
\hline Example 1 & 0.0001 & 0.0705 & 0.2877 & 0.0011 & 0.1799 & 1.5191 \\
\hline Example 2 & 0.0001 & 0.0694 & 0.7106 & 0.0039 & 0.1549 & 1.5191 \\
\hline Example 3 & 0.0001 & 0.0669 & 0.4243 & 0.0028 & 0.1694 & 1.5191 \\
\hline Example 4 & 0.0001 & 0.0962 & 0.6557 & 0.0011 & 0.2384 & 1.1667 \\
\hline Example 5 & 0.0001 & 0.0820 & 0.6459 & 0.0013 & 0.1630 & 1.1667 \\
\hline \hline
\end{tabular}

example above (4,780 inductors) were EM simulated and the errors of the surrogate models evaluated. The results are shown in Table VII. It can be checked in both tables that even the worst error in an inductor used in any of the examples
TABLE VIII

CPU TIME FOR THE DIFFERENT EXPERIMENTAL EXAMPLES.

\begin{tabular}{ccc}
\hline \hline Experiments & Offline time (h) & Online time (h) \\
\hline Inductor POF & 0.16 & - \\
\hline Example 1 & 1.23 & 6.15 \\
\hline Example 2 & 1.24 & 6.10 \\
\hline Example 3 & 1.49 & 34.5 \\
\hline Example 4 & 8.64 & 35.3 \\
\hline Example 5 & 8.84 & 35.4 \\
\hline \hline
\end{tabular}

above is quite low and, hence, the error is not expected to cause a significant deviation of the optimization process.

The efficiency of both strategies is also a very important subject. It is clear that the efficiency is different due to the inductor optimization being done either online or offline. Therefore, the time necessary for each experiment is depicted in Table VIII. The offline methodology is much more efficient due to the fact that the inductors' POF is only performed once and independently from each circuit optimization. Let us consider the following example in order to understand the time differences between both strategies: in example 4 with the online strategy, the optimization was performed with 1,000 individuals and 300 generations. Each LNA individual has 4 inductors (that are analyzed in 10 frequency points with 8 different models, in order to calculate each S-parameter component in Table I). If we multiply all the previous values, we get the astonishing value of 96 million models evaluations, whereas, in the offline strategy, the model is not used in the circuit optimization. Therefore, it is easy to understand the time differences. All optimizations were performed in a computer with two 6-core Intel ${ }^{\circ}$ E5-2639 v2 processors at $2.60 \mathrm{GHz}$.

\section{CONCLUSIONS}

In this work, a comparison was made between two different automated RF circuit design methodologies: a more traditional approach where passive components are designed during the circuit optimization stage, and another approach where inductors are optimized prior to any circuit optimization stage. It can be concluded by the comparisons performed for the design of an LNA that the offline bottom-up methodology brings advantages in both solution quality and efficiency.

\section{REFERENCES}

[1] G.E. Gielen, "CAD tools for embedded analogue circuits in mixedsignal integrated systems on chip," IEE Proceedings - Computers and Digital Techniques, vol. 152, no. 3, pp. 317-332, 2005.

[2] B. Liu, G. Gielen and F.V. Fernandez, Automated design of analog and high-frequency circuits. Springer, 2014. 
[3] Y. Xu, K.L. Hsiung, X. Li, L.T. Pileggi, S.P. Boyd, "Regular analog/RF integrated circuits design using optimization with recourse including ellipsoidal uncertainty", IEEE Trans. Comp.-Aided Design Integr. Circuits Sys., vol. 28, no. 5, pp. 623-637, 2008.

[4] A. Nieuwoudt, T. Ragheb, Y. Massoud, "Hierarchical optimization methodology for wideband low noise amplifiers", in Proc. Asia and South Pacific Design Automation Conf., pp. 68-73, 2007.

[5] J. Crols, S. Donnay, M. Steyaert and G. Gielen, "A high-level design and optimization tool for analog RF receiver front-ends," in Proc. IEEE Inter. Conf. Computer Aided Design, 1995, pp. 550-553.

[6] R.A. Rutenbar, G.E. Gielen and J. Roychowdhury, "Hierarchical modeling, optimization, and synthesis for system-level analog and RF designs," Proc. IEEE, vol. 95, no. 3, pp. 640-669, 2007.

[7] C. De Ranter, G. Van der Plas, M. Steyaert, G. Gielen, W. Sansen, "CYCLONE: automated design and layout of RF LC-oscillators", IEEE Trans. Comp.-Aided Des. Integ. Cir. Sys., vol. 21, no. 10, pp. 11611170, 2002.

[8] P. Vancorenland, C. De Ranter, M. Steyaert, G. Gielen, "Optimal RF design using smart evolutionary algorithms", in Proc. Design Automation Conf., pp. 7-10, 2000.

[9] G. Tulunay, S. Balkir, "A synthesis tool for CMOS RF low-noise amplifiers", IEEE Trans. Comp.-Aided Design Integr. Circuits Sys., vol. 27, no. 5, pp. 977-982, 2008.

[10] R. Povoa, I. Bastos, N. Lourenço, N. Horta, "Automatic synthesis of RF front-end blocks using multi-objective evolutionary techniques", Integration, the VLSI Journal, vol. 52, pp. 243-252, 2016.

[11] R. Gonzalez-Echevarria et al., "An automated design methodology of RF circuits by using pareto-optimal fronts of EM-simulated inductors," IEEE Trans. Comp.-Aided Design Integr. Circuits Sys., vol. 36, no. 1, pp. 15-26, Jan. 2017.

[12] F. Passos et al., "Physical vs surrogate models of passive RF devices", in Proc. IEEE Int. Symp. Circuits Sys., 2015, pp. 117-120.

[13] F. Passos, E. Roca, R. Castro-Lopez, F.V. Fernandez, "Radio-frequency inductor synthesis using evolutionary computation and Gaussian-process surrogate modeling," Applied Soft Computing, vol. 60, pp. 495-507, Nov. 2017

[14] M.B. Yelten, T. Zhu, S. Koziel, P.D. Franzon and M. B. Steer, "Demystifying surrogate modeling for circuits and systems," IEEE Circuits Syst. Mag, vol. 12, no. 1, pp. 45-63, 2012.

[15] F. Passos, R. Gonzalez-Echevarria, E. Roca, R. Castro-Lopez and F.V. Fernandez, "A two-step surrogate modeling strategy for single-objective and multi-objective optimization of radiofrequency circuits," Soft Computing, doi. 10.1007/s00500-018-3150-9

[16] F. Passos et al., "Enhanced systematic design of a voltage controlled oscillator using a two-step optimization methodology," Integration, the VLSI Journal, doi: 10.1016/j.vlsi.2018.02.005.

[17] K. Deb, A. Pratap, S. Agarwal, T. Meyarivan, "A fast and elitist multiobjective genetic algorithm: NSGA-II", IEEE Trans. Evol. Comp., vol. 6, no. 2, pp. 182-197, 2002.

[18] S.K. Mandal, S. Sural, A. Patra, "ANN- and PSO-based synthesis of onchip spiral inductors for RF ICs", IEEE Trans. Comp.-Aided Design Integr. Circuits Sys., vol. 27, no. 1, pp. 188-192, 2008.

[19] B. Liu, D. Zhao, P. Reynaert, G. Gielen, "Synthesis of integrated passive components for high-frequency RF ICs based on evolutionary computation and machine learning techniques", IEEE Trans. Comp.Aided Design Integr. Circuits Sys, vol. 30, no. 10, pp. 1458-1468, 2010.

[20] F. Passos, R. Gonzalez-Echevarria, E. Roca, R. Castro-Lopez and F.V. Fernandez, "Accurate synthesis of integrated RF passive components using surrogate models," in Proc. Design, Automation \& Test in Europe Conf., 2016, pp. 397-402.

[21] J.P.C. Kleijnen, "Kriging metamodeling in simulation: A review," Eur. J. of Oper. Res., vol. 192, no. 3, 2009.

[22] Cadence SpectreRF circuit simulator https://www.cadence.com /content/cadence-www/global/en_US/home/ tools/custom-ic-analog-rfdesign/circuit-simulation/spectre-rf-option.html [accessed, June 2018].

[23] R. Gonzalez-Echevarria et al., "Automated generation of the optimal performance trade-offs of integrated inductors", IEEE Trans. Comp.Aided Design Integr. Circuits Sys, vol. 33, no. 8, pp. 1269-1273, 2014.

[24] K. Okada, K. Masu, "Modeling of spiral inductors", Advanced Microwave Circuits and Systems, InTech, 2010.

[25] E. Zitzler and L. Thiele, "Multiobjective evolutionary algorithms: a comparative case study and the strength Pareto approach," IEEE Trans. Evol. Comp., vol. 3, no. 4, pp. 257-271, 1999.

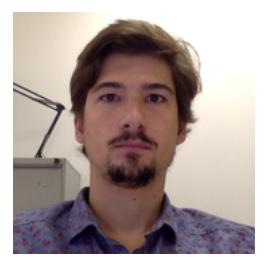

Fábio Passos received the M.Sc. degree from the New University of Lisbon, in 2013 and the Ph.D. degree from University of Seville in 2018. He is working as a postdoc researcher at the Institute of Microelectronics of Seville (IMSE-CNM, CSIC). $\mathrm{He}$ received an outstanding paper award in MIXDES 2013, was the SMACD 2016 EDA competition winner and won the SMACD 2018 best paper award. His research interests include the modeling of passive RF devices, automated design methodologies for analog/RF ICs and reliability issues in analog/RF ICs.

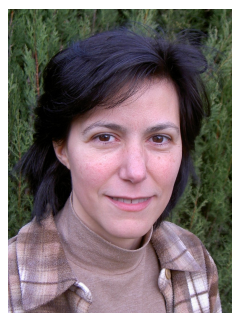

Elisenda Roca received the Ph.D. degree in Physics from the University of Barcelona, Spain, in 1995. From November 1990 to April 1995, she worked at IMEC, Leuven, Belgium. Since 1995, she has been with the Institute of Microelectronics of Seville, (IMSE-CNMCSIC), Spain, where she is a Tenured Scientist. Her research interests lie in the field of modeling and design methodologies for analog, mixed-signal and $\mathrm{RF}$ integrated circuits. She has been involved in several research projects with different institutions: CEC, ESA, ONR-NICOP, etc. She has also co-authored more than 100 papers in international journals, books, and conference proceedings.

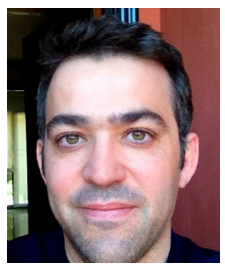

Rafael Castro-López received the M.S. degree on Electronic Physics and Ph.D. degree from the University of Seville, Spain, in 1998 and 2005, respectively. Since 1998, he has been working at the Institute of Microelectronics of Seville (CSIC-IMSECNM) of the Spanish Microelectronics Center, where he is a Tenured Scientist. His research interests lie in the field of integrated circuits, especially design and computer-aided design for analog and mixed-signal circuits. He has participated in several national and international R\&D projects and coauthored more than 50 international scientific publications, including journals, conference papers, book chapters and the book Reuse-based Methodologies and tools in the Design of Analog and Mixed-Signal Integrated Circuits (Springer, 2006).

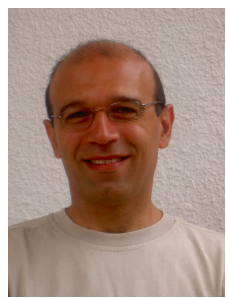

Francisco V. Fernández got the $\mathrm{Ph}$. D. degree in Microelectronics from the University of Seville, Spain, in 1992. In 1993, he worked as a postdoctoral research fellow at Katholieke Universiteit Leuven (Belgium). From 1995 to 2009, he was an Associate Professor at the Dept. of Electronics and Electromagnetism of University of Seville, where he was promoted to full professor in 2009. He is also a Department Head at IMSE-CNM (University of Seville and CSIC). Dr. Fernández was the Editor-in-Chief of Integration, the VLSI Journal (Elsevier) from 2005 to 2015. His research interests lie in the design and design methodologies of analog, mixed-signal and radiofrequency circuits, and reliable circuit design. Dr. Fernández has authored or edited five books and has co-authored more than 200 papers in international journals and conferences. He has served as General Chair of three international conferences and regularly serves at the Program Committee of several international conferences. He has also participated as researcher or main researcher in numerous national and international $R \& D$ projects. 